\section{Régénération des cellules $\beta$ pancréatiques par l'activation de la voie de signalisation GABA}

Andhira Vieira, Nouha Ben-Othman, Noémie Druelle, Monica Courtney, Fabio Avolio, Tiziana Napolitano, Elisabet Gjernes, Biljana Hadzic, Sergi Navarro Sanz, Serena Silvano, Patrick Collombat
Université Côte d'Azur, Inserm, CNRS, iBV, 28, avenue de Valrose, 06108 Nice,

France.

collombat@unice.fr
> Le pancréas est constitué de deux tissus principaux, les tissus exocrine et endocrine. Le compartiment exocrine est composé de cellules acinaires et canalaires qui, respectivement, sécrètent ou transportent des enzymes digestives dans l'intestin. Le tissu endocrine, quant à lui, est organisé en petits groupes de cellules appelés îlots de Langerhans. Ces îlots sont constitués de plusieurs types cellulaires parmi lesquels les cellules $\beta$, productrices d'insuline, une hormone qui diminue le taux de glucose sanguin en cas d'apport sucré, et les cellules $\alpha$, sécrétrices de glucagon, une hormone induisant une augmentation du taux de glucose sanguin en cas d'hypoglycémie. L'insuline et le glucagon jouent donc un rôle crucial pour le maintien de I'homéostasie glucidique.

Le diabète de type 1 est une maladie auto-immune caractérisée par la destruction sélective des cellules $\beta$. L'incapacité à produire de l'insuline qui en résulte induit une hyperglycémie chronique qui provoque une élévation de la viscosité sanguine, augmentant les risques d'atteintes rénales, de cécité, voire d'amputation. Le nombre de patients affectés par cette pathologie augmente chaque année, ce qui fait du diabète de type 1 une question de santé publique d'importance croissante.

Afin de trouver une alternative aux traitements actuels (principalement l'injection d'insuline exogène) relati- vement efficaces mais néanmoins très contraignants pour les patients, de nombreuses recherches s'attachent à trouver de nouvelles façons de (ré) générer des cellules $\beta$ sécrétant l'insuline. À cette fin, nous nous sommes intéressés aux gènes Arx (aristaless-related homeobox) et Pax4 (paired box gene 4). Nous avons démontré que, durant l'embryogenèse, le gène Arx induit la différenciation des cellules $\alpha$ alors que le gène Pax4 promeut celle des cellules $\beta[1]$. L'utilisation de souris transgéniques permettant de forcer l'expression du gène Arx dans les cellules $\beta$ adultes nous a alors permis de mettre en évidence une conversion de ces dernières en cellules $\alpha$ [2], démontrant pour la première fois in vivo la plasticité cellulaire des cellules $\beta$ adultes et suggérant ainsi qu'une conversion inverse pourrait survenir. Nous avons donc généré des animaux transgéniques permettant l'expression forcée du gène Pax4 (l'antagoniste d'Arx) ou l'inactivation d'Arx dans les cellules $\alpha$ P. Collombat et A. Mansouri, [3] $(\rightarrow)$. Dans $m / s n^{\circ} 8-9$, août-septembre les deux cas, 2009, page 763

une conversion des cellules $\alpha$ en cellules « $\beta$-similaires» (cellules présentant un phénotype de cellule $\beta$ ) a été observée [4-6] $(\rightarrow)$. Nos résultats ont également montré l'exis-

$(\rightarrow)$ Voir la Synthèse de A. Vieira, $m / s n^{\circ} 8-9$, août-septembre 2013, page 749 tence d'un cycle continu de régénération des cellules $\alpha$ (probablement pour compenser leur conversion en cellules $\beta$-similaires), celles-ci étant à nouveau converties en cellules $\beta$-similaires par l'expression forcée du gène Pax4 ou par l'inactivation d'Arx. Des résultats complémentaires ont indiqué que l'inactivation d'Arx dans les cellules $\alpha$ est en fait le déclencheur de la conversion des cellules $\alpha$ en cellules $\beta$-similaires, l'expression forcée de Pax4 n'agissant que pour inhiber Arx. Les cellules $\beta$-similaires ainsi générées apparaissent fonctionnelles et capables d'inverser les conséquences d'un diabète induit chimiquement in vivo.

Bien que très encourageants, ces résultats restent néanmoins éloignés d'une application humaine tant qu'un activateur chimique de la conversion des cellules $\alpha$ vers des cellules $\beta$-similaires n'est pas identifié. Dans ce but, nous avons procédé à des criblages moléculaires, espérant isoler un tel composé. $\varepsilon$ collaboration avec l'équipe de Stefan Kubicek en Autriche, nous avons ainsi mis en évidence deux composés distincts, l'acide $\gamma$-aminobutyrique (GABA) et l'arthéméther, qui activent la même voie de signalisation et induisent cette conversion $[7,8]$.

\section{L'acide $\boldsymbol{\gamma}$-aminobutyrique ou GABA}

Notre laboratoire a identifié l'acide $\gamma$-aminobutyrique (GABA) comme poten- 


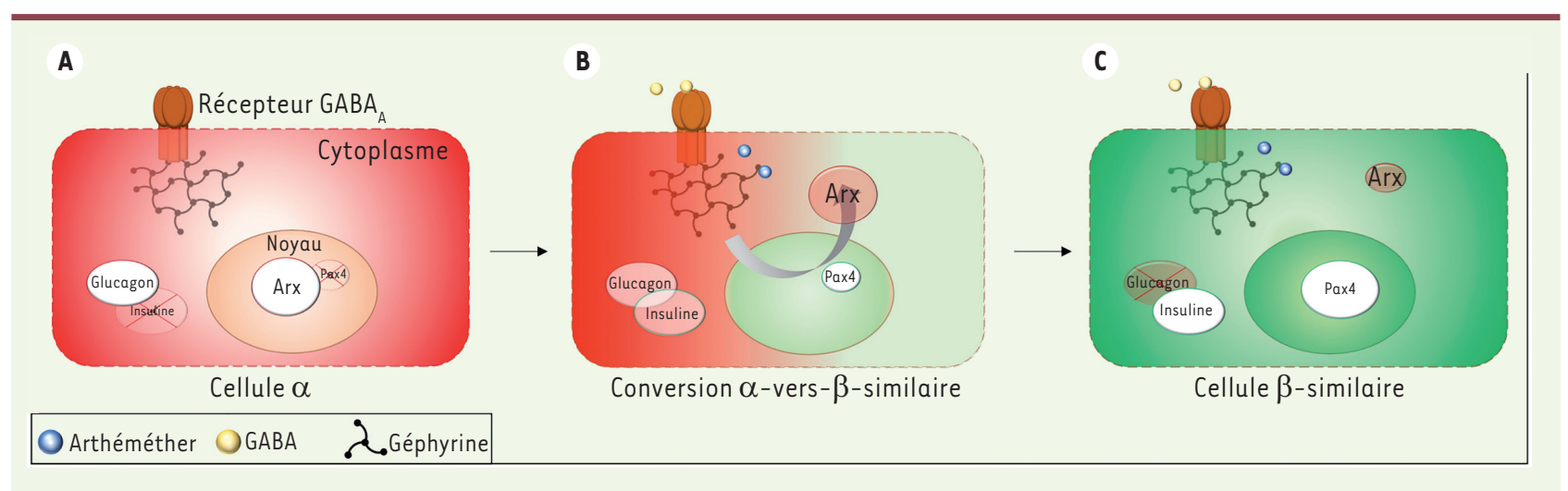

Figure 1. Conversion des cellules $\alpha$ des îlots $\boldsymbol{\beta}$ de Langerhans en cellules $\boldsymbol{\beta}$-similaires par le GABA ou l'arthéméther. $A$. Le récepteur GABA $A_{A}$, exprimé à la surface des cellules $\alpha$, interagit par son domaine cytoplasmique avec la géphyrine, une protéine d'échafaudage. Dans le noyau, le facteur de transcription Arx réprime l'expression du gène Pax4, assurant le maintien de l'identité cellulaire $\alpha$. $B$. Le GABA, en se fixant à son récepteur $G A B A_{A}$, active la transduction du signal par l'intermédiaire de la géphyrine. L'arthéméther, quant à lui, interagit directement avec la géphyrine pour activer la même voie de signalisation, les deux composés ayant finalement le même effet sur les cellules $\alpha$ : la translocation du facteur Arx hors du noyau qui l'empêche de réprimer le gène Pax4. C. L'expression ectopique de Pax4 résultante induit la conversion des cellules $\alpha$ en cellules $\beta$-similaires sécrétant de l'insuline. GABA : acide $\gamma$-aminobutyrique; Arx : aristaless-related homeobox; Pax4 : paired box gene 4.

tiel inducteur de conversion des cellules $\alpha$ en cellules $\beta$. Ce composé est un neurotransmetteur également produit par les cellules $\beta$. Nos premières analyses ont démontré que le traitement in vitro de cellules $\alpha$ avec des doses croissantes de GABA induit une diminution progressive de l'expression du gène Arx. Le GABA est donc un candidat envisageable pour l'induction de la conversion des cellules $\alpha$ en cellules $\beta$-similaires in vivo. L'analyse des pancréas de souris sauvages traitées par des injections quotidiennes de GABA a montré une remarquable augmentation du nombre et de la taille des îlots de Langerhans, avec une prépondérance notable des cellules $\beta$ sécrétrices d'insuline. Des études de traçage de lignage cellulaire (marquage permanent des cellules $\alpha$ et suivi de leur devenir) combinées à des analyses immunohistochimiques, ont démontré une conversion des cellules $\alpha$ en cellules $\beta$-similaires. Cette conversion s'accompagne d'une régénération continue des cellules $\alpha$ pour compenser leur perte, induisant une nouvelle vague de conversion en cellules sécrétrices d'insuline du fait de la présence de GABA. Ces cycles de régénération conduisent à une accumulation de cellules $\beta$-similaires présentant des caractéristiques phéno- typiques et fonctionnelles comparables à celles des cellules $\beta$ endogènes. De plus, l'ablation chimique de la population de cellules $\beta$ in vivo, engendrant normalement une hyperglycémie chronique, est contrée par les injections quotidiennes de GABA provoquant la régénération d'un pool de cellules $\beta$-similaires par conversion de cellules $\alpha[7]$. Ces résultats très encourageants peuvent s'expliquer par le mode d'action du GABA sur les cellules $\alpha$ des îlots de Langerhans, mis en évidence par nos collègues autrichiens (voir cidessous)

\section{Artéméther}

Au cours d'un criblage indépendant, l'équipe du Dr Kubicek a en effet identifié l'arthéméther, un dérivé de l'artémisinine utilisé comme traitement antipaludéen [9]

$(\rightarrow)$, comme pouvant promouvoir la sécrétion d'insuline

$(\rightarrow)$ Voir l'article Nobel de D. Mazier et M. Thellier, $m / s n^{\circ} 1$, janvier 2016, page 106 par des cellules $\alpha$ in vitro. Des analyses plus détaillées ont permis de déterminer que cette conversion des cellules $\alpha$ en cellules sécrétrices d'insuline est possible grâce à la stabilisation de la géphyrine, une protéine interagissant avec le récepteur du GABA, GABA , ce qui conduit alors à une potentialisation de la voie de signalisation GABA. Cette suractivation entraîne la translocation d'Arx hors du noyau cellulaire, empêchant son activité transcriptionnelle. Ce déplacement vers le cytoplasme équivaut à une inactivation et peut expliquer que le même phénotype soit obtenu lorsque le gène Arx est directement inactivé dans les cellules $\alpha$, à savoir une conversion des cellules $\alpha$ en cellules $\beta$-similaires [8].

\section{En conclusion}

Ces résultats prometteurs mettent en évidence deux nouvelles classes de molécules pour restaurer un nombre suffisant de cellules $\beta$ chez les patients atteints de diabète de type 1 , les stimulateurs de la voie de signalisation GABA et les stabilisateurs de géphyrine (Figure 1). Les deux composés qui ont été identifiés (GABA et arthéméter) ont déjà donné des résultats prometteurs sur les îlots de Langerhans humains in vitro et ex vivo $[7,8]$. Bien que des études complémentaires soient évidemment nécessaires pour mieux comprendre leurs mécanismes d'action avant d'envisager une application chez l'homme, 
le fait que le GABA et l'arthéméther soient déjà commercialisés accélérera considérablement l'éventuelle mise en place d'un traitement. $\diamond$

Induction of pancreatic $\beta$-like cell regeneration by activation of GABA signaling pathways

\section{LIENS D'INTÉRÊT}

Les auteurs déclarent n'avoir aucun lien d'intérêt concernant les données publiées dans cet article.

\section{RÉFÉRENCES}

1. Collombat P, Hecksher-Sørensen J, Serup P, et al. Specifying pancreatic endocrine cell fates. Mech Dev 2006; 123: 501-12.

2. Collombat P, Hecksher-Sørensen J, Krull J, et al. Embryonic endocrine pancreas and mature $\beta$ cells acquire $\alpha$ and PP cell phenotypes upon Arx misexpression. J Clin Invest 2007 ; 117 : 961-70.

3. Collombat P, Mansouri A. Conversion de cellules $\alpha$ pancréatiques en cellules $\beta$. Med Sci (Paris) 2009 ; $25: 763-6$.

4. Al-Hasani K, Pfeifer A, Courtney M, et al. Adult ductlining cells can reprogram into $\beta$-like cells able to counter repeated cycles of toxin-induced diabetes. Dev Cell $2013 ; 26: 86-100$.
5. Courtney M, Gjernes $\varepsilon$, Druelle N, et al. The inactivation of arx in pancreatic $\alpha$-cells triggers their neogenesis and conversion into functional $\beta$-like cells. PLoS Genet 2013 ; 9 : e1003934.

6. Vieira A, Druelle N, Courtney M, et al. Reprogrammation des cellules pancréatiques en cellules $\beta$. Med Sci (Paris) 2013 ; $29: 749-55$.

7. Ben-Othman N, Vieira A, Courtney M, et al. Long-term GABA administration induces alpha cell-mediated beta-like cell neogenesis. Cell 2017 ; 168 : 73-85.ell.

8. Li J, Casteels T, Frogne T, et al. Artemisinins target GABAA receptor signaling and impair $\alpha$ cell identity. Cell 2017 ; $168: 86$-100.el5.

9. Mazier D, Thellier M. Youyou Tu, de Mao Zedong au Prix Nobel. Med Sci (Paris) 2016 ; 32 : 106-9.

\section{NOUVELle}

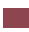 \\ Le virus d'Epstein-Barr et le centriole, nouvelles liaisons dangereuses}

Henri-Jacques Delecluse, Susanne Fink, Ming-Han Tsai, Anatoliy Shumilov

> Le virus d'Epstein-Barr (VEB) est un membre de la famille des virus herpès qui infecte la majorité de la population [1]. Le premier contact avec le virus s'effectue en général dans les premiers mois après la naissance et est soit asymptomatique, soit accompagné d'une pharyngite. On estime que plus de $90 \%$ de la population adulte est infectée [1]. Le virus persiste dans l'organisme infecté jusqu'à la mort de son hôte. II s'y multiplie régulièrement pour induire la production de nouvelles particules virales principalement retrouvées dans la salive. Celle-ci véhicule les virus lors d'un contact rapproché interindividuel [1]. Dans les populations où les contacts sociaux sont plus tardifs, I'infection par le virus d'Epstein-Barr est souvent retardée jusqu'à l'adolescence, provoquant, dans un tiers des cas, une mononucléose infectieuse, un syndrome accompagné d'une pharyngite, de fièvre et d'une lymphadé- nopathie ${ }^{1}[1]$. Ces signes cliniques sont en général transitoires, même s'ils peuvent être suivis d'une fatigue persistante. Cette maladie, en apparence anodine ne doit cependant pas faire oublier que le virus d'EpsteinBarr est un virus oncogène [2]. Ce virus a en effet été découvert dans un cancer du système lymphatique, le lymphome de Burkitt, du nom du chirurgien qui l'a décrit. Le développement des techniques de greffes d'organes ou de moelle osseuse et le traitement immunosuppresseur qu'elles nécessitent, ont souligné le pouvoir oncogène de ce virus par ailleurs commun. En l'absence d'un système immunitaire fonctionnel, le virus induit une prolifération illimitée des lymphocytes B, sa cible favorite, qui se traduit cliniquement par le développement de lymphomes dits « du

${ }^{1}$ Une lymphadénopathie est une hypertrophie des ganglions lymphatiques.
Unité Inserm/Deutsches

krebsforschungszentrum (DKFZ) 1074, 69120 Heidelberg, Allemagne.

h.delecluse@dkfz.de

transplanté » $[1,2]$. De nombreuses études épidémiologiques indiquent que la mononucléose infectieuse a parfois des conséquences graves comme l'apparition d'un lymphome de Hodgkin ${ }^{2}[1,2]$. Le spectre des cancers induits par le virus d'EpsteinBarr est plus étendu : il est bien établi que le virus est associé au cancer du nasopharynx, particulièrement fréquent en Asie du sud-est et dans une moindre mesure en Afrique du nord [2, 3] $(\rightarrow)$. Le virus d'Epstein-Barr

$\rightarrow \rightarrow$ Voir la Synthèse de P. Busson et al., $m / s n^{\circ} 4$, avril 2004, page 453

contribue aussi au développement d'un type particulier de cancer de l'estomac [2].

Si la responsabilité du virus dans le développement de ces cancers est maintenant bien établie, les mécanismes

\footnotetext{
${ }^{2}$ Ce type de lymphome regroupe les cancers qui apparaissent dans les ganglions lymphatiques et se propagent ensuite aux régions voisines.
} 